\title{
Association of body pain and chronic disease: evidence from a 7-year population-based study in China
}

\author{
Yaxin Luo, ${ }^{1}$ Zheran Liu, ${ }^{2}$ Lianlian Yang, ${ }^{2}$ Juejin Li, ${ }^{3}$ Qiang Zhang, ${ }^{4}$ Xingchen Peng, ${ }^{2}$ \\ Xiaolin $\mathrm{Hu}^{3}$
}

- Additional supplemental material is published online only. To view, please visit the journal online (http://dx.doi.org/ 10.1136/rapm-2021-102700).

For numbered affiliations see end of article.

\section{Correspondence to \\ Xiaolin Hu; \\ huxiaolin1220@126.com \\ Dr Xingchen Peng; \\ pxx2014@163.com \\ Dr Qiang Zhang; \\ qiangzhang@scu.edu.cn}

YL and ZL contributed equally.

YL and ZL are joint first authors.

Received 12 March 2021

Accepted 26 May 2021

Published Online First

7 June 2021

\section{Linked}

- http://dx.doi.org/10.1136/ rapm-2021-102949

Check for updates

(C) American Society of Regional Anesthesia \& Pain Medicine 2021. No commercial re-use. See rights and permissions. Published by BMJ.

To cite: Luo Y, Liu Z, Yang L, et al. Reg Anesth Pain Med 2021:46:745-751.

\begin{abstract}
Background Evidence is limited on the risk impact of body pain on future chronic disease. The present study aimed to investigate the association between body pain and chronic diseases.

Methods Data were analyzed using four waves of the China Health and Retirement Longitudinal Study with 17 708 individual respondents aged 45 years and older. The association between body pain and chronic disease was estimated in both a cross-sectional cohort (2011) and a longitudinal cohort (2011-2018). The key outcomes include the incidence of overall and any specific chronic diseases. The associations among different body pain sites and 10 independent chronic disease risks were also assessed.
\end{abstract}

Findings A total of 17128 participants in 2011 were included in the cross-sectional cohort and 5611 participants were included in the 2011-2018 longitudinal cohort. Body pain showed an association with overall chronic disease in both the cross-sectional models (OR 2.71, 95\% Cl 2.47 to 2.98) and longitudinal model (risk ratio (RR) $1.21,95 \% \mathrm{Cl} 1.07$ to 1.35 ). Moreover, body pain was found to be associated with an increased risk of chronic respiratory disease (RR 1.43, $95 \% \mathrm{Cl} 1.06$ to 1.92), heart disease (RR $1.45,95 \% \mathrm{Cl}$ 1.12 to 1.89 ), kidney disease (RR $1.83,95 \% \mathrm{Cl} 1.28$ to 2.6), and digestive disease (RR $1.48,95 \% \mathrm{Cl} 1.17$ to 1.88).

Conclusion Body pain is associated with major disease and mortality. Future clinical research should be targeted to whether or not improved pain control can mitigate this population-level disease burden.

\section{INTRODUCTION}

Body pain is a global problem associated with both poor psychological and physical health. ${ }^{1}$ The International Association for the Study of Pain has defined pain as 'an unpleasant sensory and emotional experience associated with or resembling that associated with actual or potential tissue damage'. ${ }^{2}$ Such experience could be caused by multiple factors, including sociodemographic, lifestyle, clinical, and psychological factors. ${ }^{3}$ According to the Global Burden of Disease Study 2016, pain, especially low back pain and migraine, and their related diseases are the leading causes of disability and disease burden. ${ }^{4}$ At present, chronic pain conditions have been reconsidered as a distinct disease entity rather than a simple description of symptoms. ${ }^{5}$
Associations have been found between chronic body pain and mortality. Torrance et al found that severe chronic pain is associated with all-cause mortality and cardiovascular-specific mortality. ${ }^{6}$ According to results from half a million prospective cohorts and meta-analyses, chronic widespread pain is related to cancer, cardiovascular, respiratory, and other disease-related causes of death. Although body pain has now been widely linked to current disease states, examination of the relationship between body pain and future disease risk is relatively rare. One meta-analysis has reported that neck or back pain could greatly increase the future risk of chronic disease, particularly in cardiovascular disease. ${ }^{8}$ For site-specific pain, the study also reported that lower back pain in women could greatly increase the future diabetes risk in a subsequent 11-year interval, however, in the man cohort, this association disappeared. ${ }^{9}$

Although a suggested link has been discovered between body pain and chronic disease, to the best of our knowledge, a direct longitudinal prospective study is still warranted to add solid evidence on this association. The purpose of the present study was to examine the relationship between body pain and the main chronic disease using a large prospective cohort in a Chinese population.

\section{METHODS}

\section{Study design and cohort selection}

The analysis was based on the four waves of the China Health and Retirement Longitudinal Study (CHARLS), which began in 2011 (wave 1) and was followed up in 2013 (wave 2), 2015 (wave 3), and 2018 (wave 4). The details of the study design and population characteristics have been reported previously. ${ }^{10}$ Briefly, using multistage stratified probability proportionate to size sampling, CHARLS collected nationally representative samples comprizing 17708 individual respondents aged 45 years and older between June 2011 and March 2012. The baseline survey was conducted and had a response rate of $80.5 \%$.

Participants with complete body pain and doctordiagnosed chronic disease in wave 1 were included in the cross-sectional study. After participants with missing data $(n=594)$ were excluded, 17128 participants in wave 1 were included. The association between body pain and the existing chronic disease status was estimated among the included participants. 
Participants with data across four waves from 2011 to 2018 were included in the longitudinal cohort. They were excluded if they (1) were missing data on either body pain or chronic disease $(n=594)$ or (2) had any previous self-reported chronic disease at baseline $(\mathrm{n}=11$ 486). Ultimately, 5628 participants were included. Overall and sitespecific body pain, as the main exposure, were obtained from wave 1 and the subsequent chronic disease development, as the main outcome, was obtained from waves 2 to 4 .

\section{Ascertainment of exposure}

For each wave, information on participants was collected through one-to-one interviews with a structured questionnaire. One question was: 'Are you often troubled with any body pains?' Any participant who answered 'yes' was then given the following question: 'On what part of your body do you feel pain? Please list all parts of the body which are currently feeling pain (You can select more than one answer)' with several body regions given as options ('head', 'shoulder', 'arm', 'wrist', 'fingers', 'chest', 'stomach', 'back', 'waist', 'buttocks', 'leg', 'knees', 'ankle', 'toes', and 'neck'). Based on previous evidence, we further recategorized the body region into head, neck/ shoulders, arms/hands, frontal torso/genitals, back, buttocks, upper legs, and lower legs/feet. ${ }^{11}$ Multiple sites of pain were defined as pain site numbers larger than 1 .

\section{Ascertainment of outcomes}

The main outcome of the present study included any doctordiagnosed disease reported by participants identified from waves 2 to 4 . These doctor-diagnosed diseases included hypertension, dyslipidemia, diabetes or high blood sugar, cancer or malignant tumor (excluding minor skin cancers), chronic lung diseases, such as chronic bronchitis, emphysema (excluding tumors, or cancer), liver disease (except fatty liver, tumors, and cancer), heart disease (including heart attack, coronary heart disease, angina, congestive heart failure, or other heart problems), stroke, kidney disease (except for tumor or cancer), stomach or other digestive diseases (except for tumor or cancer), and asthma. Among them, chronic lung diseases and asthma were recategorized as respiratory diseases. Mortality information was acquired from the Exit and Verbal Autopsy Questionnaire in wave 2, on which the death date was acquired from death certificates, medical records, or interviews with the relatives of participants.

\section{Ascertainment of covariates}

Information on potential confounders was acquired from the baseline. Age at recruitment was categorized into 10-year intervals. Educational qualification was categorized as illiterate, primary school or lower, middle school, high school, or higher. Marital status was categorized as married, single/other, or widowed. Body mass index (BMI) was computed in $\mathrm{kg}^{2} / \mathrm{m}$ and subdivided into three groups: $\leq 24.9,25-29.9$, and $\geq 30$. Restless sleep times were categorized as rare, some or a little, occasionally, most, or all. Difficulty with running or jogging approximately $1 \mathrm{~km}$ was categorized as no, have difficulty, cannot, and need help. Smoking status and alcohol status were ascertained.

\section{Statistical analyses}

All analyses were conducted with R V.3.6.3 (R Development Core Team, Vienna, Austria), and a two-sided $\mathrm{p}<0.05$ was set as statistically significant. In the cross-sectional analysis, the association between body pain and chronic disease was estimated through ORs, with $95 \%$ CIs, with data from 2011 by logistic regression. For participants without previous chronic diseases at baseline, the association between the pain and the incidence of chronic diseases was evaluated through risk ratios (RRs) using a generalized linear model (with a log link and Poisson distribution). Three models were constructed according to the adjustment degree. Model 1 was a univariate model for body pain. Model 2 was additionally adjusted for the inherent characteristics, including gender, age at recruitment, marital status, and educational level. Model 3 was further adjusted for potential lifestyle and health-related confounders, including BMI, sleep quality, difficulty in activity, smoking status, and alcohol consumption status. A Cox proportional hazards model was used to investigate the association of body pain and mortality, and the results are reported as HRs together with 95\% CIs. Follow-up time in months from baseline was used as the timescale, and for each participant, the end of follow-up was set at date of death or date of interview of wave 2, whichever came first. Participants who did not take part in the interview of wave 2 were excluded from the analysis of mortality. Model 3 was not applied to adjust the all-cause mortality, considering nearly $38.8 \%(153 / 394)$ of death events missing at least one lifestyle covariate included in model 3.

Potential interactions among all potential risk factors were estimated, including age ( $<65$ or $\geq 65$ years), gender (male or female), educational level ((middle school or lower) or (high school or higher)), marital status (married or single/widow/other), BMI $(<25$ or $\geq 25$ ), smoking status (never or current/ever), alcohol consumption status (never or current/ever), restless sleep times (rare or more than once a week), and difficulty running $1 \mathrm{~km}$ (no or yes). In sensitivity analyses, we excluded participants who developed any chronic disease within the first 2 years of follow-up to avoid reverse causality. Furthermore, we reconducted the association analysis by restricting the participants to a shorter follow-up time. Last, we repeated the analyses by using log-binomial regression rather than modified Poisson regression to evaluate RR with 95\% CI.

\section{RESULTS}

\section{Population characteristics}

As presented in table 1, in wave 1 of CHARLS, 17128 participants with complete information were ultimately included in the cross-sectional cohort. Among them, 5544 (32.37\%) participants with body pain and 11584 (67.63\%) participants without body pain were identified. Compared with participants without body pain, participants troubled with body pain were more likely to be female and widowed with lower education levels, had difficulty in running $1 \mathrm{~km}$, had sleeplessness, and never smoke or consumed alcohol. In the longitudinal cohort, the baseline characteristics of a total of 5628 qualified participants were included according to the inclusion and exclusion criteria (table 1). Among them, 4711 (83.71\%) participants reported no current experience of body pain and 917 (16.29\%) participants reported trouble with body pain. The population distribution in the longitudinal cohort was similar to that in the cross-sectional cohort. Generally, compared with participants who were included in the longitudinal cohort, participants who were excluded from the longitudinal cohort were more likely to report body pain (online supplemental table S1).

\section{The association between body pain and overall chronic disease}

In a cross-sectional setting of analyzed participants in wave 1 , as presented in table 2, strong associations between body pain and overall chronic disease were found (model 3: OR 2.71, 95\% CI 2.47 to 2.98 ). Further analyses showed that specific body pain was found widely associated with most independent chronic diseases (online supplemental figure S1) at baseline. 


\section{Original research}

Table 1 The baseline characteristics of participants stratified by body pain in the cross-sectional cohort and longitudinal cohort

\begin{tabular}{|c|c|c|c|c|c|c|}
\hline & Cross-sectiona & $t(\mathrm{~N}=17$ 128) & & Longitudinal c & $\mathrm{N}=5628)$ & \\
\hline & Without pain & With pain & P value* & Without pain & With pain & $P$ value \\
\hline Sample size, $\mathrm{n}$ & 11584 & 5544 & & 4711 & 917 & \\
\hline Age (years), n (\%) & & & & & & \\
\hline $45-54$ & $4256(36.7)$ & $1767(31.9)$ & $<0.001$ & $2135(45.3)$ & $364(39.7)$ & $<0.01$ \\
\hline $55-64$ & 4249 (36.7) & $2143(38.7)$ & & 1619 (34.4) & $321(35.0)$ & \\
\hline $65-74$ & 2065 (17.8) & $1129(20.4)$ & & $632(13.4)$ & $145(15.8)$ & \\
\hline$\geq 75$ & $1014(8.8)$ & $505(9.1)$ & & $325(6.9)$ & $87(9.5)$ & \\
\hline Sex, n (\%) & & & & & & \\
\hline Male & $6163(53.2)$ & 2185 (39.4) & $<0.001$ & $2543(54.0)$ & $367(40.0)$ & $<0.001$ \\
\hline Female & $5413(46.7)$ & $3355(60.5)$ & & $2166(46.0)$ & $550(60.0)$ & \\
\hline Missing & $8(0.1)$ & $4(0.1)$ & & $2(0)$ & $0(0)$ & \\
\hline Education level, n (\%) & & & & & & \\
\hline Illiterate & $2782(24.0)$ & 1889 (34.1) & $<0.001$ & $1081(22.9)$ & $331(36.1)$ & $<0.001$ \\
\hline Primary school and lower & 4336 (37.4) & $2378(42.9)$ & & $1702(36.1)$ & $365(39.8)$ & \\
\hline Middle school & $2666(23.0)$ & $874(15.8)$ & & $1173(24.9)$ & $150(16.4)$ & \\
\hline High school and higher & 1785 (15.4) & $398(7.2)$ & & 747 (15.9) & $70(7.6)$ & \\
\hline Missing & $15(0.1)$ & $5(0.1)$ & & $8(0.2)$ & $1(0.1)$ & \\
\hline Marital status, $\mathrm{n}(\%)$ & & & & & & \\
\hline Married & $10192(88.0)$ & $4722(85.2)$ & $<0.001$ & $4196(89.1)$ & $788(85.9)$ & 0.01 \\
\hline Single/Other & $255(2.2)$ & $126(2.3)$ & & $124(2.6)$ & $26(2.8)$ & \\
\hline Widowed & $1137(9.8)$ & $695(12.5)$ & & $391(8.3)$ & $103(11.2)$ & \\
\hline Missing & $0(0)$ & $1(0)$ & & $0(0)$ & $0(0)$ & \\
\hline BMI, n (\%) & & & & & & \\
\hline$\leq 24.9$ & $6981(60.3)$ & $3464(62.5)$ & 0.11 & $3027(64.3)$ & $643(70.1)$ & 0.04 \\
\hline $25-29.9$ & 2995 (25.9) & $1389(25.1)$ & & $1070(22.7)$ & $175(19.1)$ & \\
\hline $30-34.9$ & $453(3.9)$ & $237(4.3)$ & & $120(2.5)$ & $25(2.7)$ & \\
\hline$\geq 35$ & $63(0.5)$ & $41(0.7)$ & & $19(0.4)$ & $4(0.4)$ & \\
\hline Missing & $1092(9.4)$ & $413(7.4)$ & & $475(10.1)$ & $70(7.6)$ & \\
\hline Alcohol consumption status, & & & & & & \\
\hline Current or former & $4116(35.5)$ & $1583(28.6)$ & $<0.001$ & $1965(41.7)$ & $326(35.6)$ & $<0.001$ \\
\hline Never & $7456(64.4)$ & 3958 (71.4) & & $2744(58.2)$ & $591(64.4)$ & \\
\hline Missing & $12(0.1)$ & $3(0.1)$ & & $2(0)$ & $0(0)$ & \\
\hline Smoking status, n (\%) & & & & & & \\
\hline Yes & $4882(42.1)$ & $1990(35.9)$ & $<0.001$ & $1811(38.4)$ & $308(33.6)$ & $<0.01$ \\
\hline No & $6695(57.8)$ & $3551(64.1)$ & & $2895(61.5)$ & $609(66.4)$ & \\
\hline Missing & $7(0.1)$ & $3(0.1)$ & & $5(0.1)$ & $0(0)$ & \\
\hline Restless sleep times, n (\%) & & & & & & \\
\hline Rare & $6003(51.8)$ & $1609(29.0)$ & $<0.001$ & $2666(56.6)$ & $319(34.8)$ & $<0.001$ \\
\hline Some or a little & $1764(15.2)$ & $861(15.5)$ & & $655(13.9)$ & $147(16.0)$ & \\
\hline Occasionally & $1311(11.3)$ & $1020(18.4)$ & & $430(9.1)$ & $149(16.2)$ & \\
\hline Most or all & $1465(12.6)$ & $1599(28.8)$ & & $467(9.9)$ & $212(23.1)$ & \\
\hline Missing & $1041(9.0)$ & $455(8.2)$ & & $493(10.5)$ & $90(9.8)$ & \\
\hline Difficulty with running $1 \mathrm{~km}$, & & & & & & \\
\hline Cannot do or need help & 3689 (31.8) & $3323(59.9)$ & $<0.001$ & $997(21.2)$ & $421(45.9)$ & $<0.001$ \\
\hline Have difficult & $1174(10.1)$ & $596(10.8)$ & & $431(9.1)$ & $104(11.3)$ & \\
\hline No & $6491(56.0)$ & $1545(27.9)$ & & $3183(67.6)$ & $372(40.6)$ & \\
\hline Missing & $230(2.0)$ & $80(1.4)$ & & $100(2.1)$ & $20(2.2)$ & \\
\hline
\end{tabular}

* $\mathrm{P}$ value was calculated to compare the difference between participants without and without body pain in the cross-sectional and longitudinal cohort using $\chi^{2}$ test. BMI, body mass index.

As presented in table 2, associations were also found in the longitudinal cohort between body pain and overall chronic disease (model 3: $\mathrm{RR}=1.21$, 95\% CI 1.07 to 1.35 ). With multiple sites of body pain (number of pain sites $>1$ ), the risk of chronic disease increased $\left(\mathrm{P}_{\text {trend }}=0.004\right.$, online supplemental table S2). Body pain with multiple sites elevated the future risk of chronic disease by $23 \%$ (model 3: RR 1.23, 95\% CI 1.07 to 1.41). The relationship between different pain sites and chronic disease was also tested. We found that chronic diseases risk was particularly elevated in participants with neck/shoulder pain (RR 1.27, 95\% CI 1.07 to 1.5 ) and back pain (RR 1.27, 95\% CI 1.11 to 1.46 , online supplemental table S3). 
Table 2 Unadjusted models and adjusted models estimated the association between body pain and chronic disease in the cross-sectional cohort and longitudinal cohort

\begin{tabular}{|c|c|c|c|c|c|c|}
\hline & \multicolumn{3}{|c|}{ Cross-sectional cohort (2011) } & \multicolumn{3}{|c|}{ Longitudinal cohort (2011-2018) } \\
\hline & No. of participants & OR $(95 \% \mathrm{Cl})$ & $P$ value & No. of participants & RR $(95 \% \mathrm{Cl})$ & $P$ value \\
\hline Model $1^{*}$ & 17114 & 3.45 (3.19 to 3.74$)$ & $<0.001$ & 5263 & 1.25 (1.12 to 1.38$)$ & $<0.001$ \\
\hline Model $2 \dagger$ & 17082 & 3.43 (3.16 to 3.72 ) & $<0.001$ & 5253 & 1.24 (1.12 to 1.38$)$ & $<0.01$ \\
\hline Model 3‡ & 14471 & 2.71 (2.47 to 2.98 ) & $<0.001$ & 4483 & 1.21 (1.07 to 1.35$)$ & $<0.01$ \\
\hline
\end{tabular}

*Univariate model.

†Additionally adjusted for gender, age at recruitment, marital status, and educational level.

$\ddagger$ Additionally adjusted for BMI, sleep quality, difficulty in activity, smoking status, and alcohol consumption status.

$\mathrm{BMI}$, body mass index; RR, risk ratio.

\section{The association between pain sites and specific chronic diseases}

As presented in table 3, body pain was associated with an increased risk of chronic respiratory disease (RR 1.43, 95\% CI 1.06 to 1.92 ), heart disease (RR $1.45,95 \%$ CI 1.12 to 1.89 ), kidney disease (RR 1.83 , 95\% CI 1.28 to 2.6 ), and digestive disease (RR 1.48, 95\% CI 1.17 to 1.88 ).

Furthermore, as presented in figure 1, when comparing participants with site-specific pain versus without corresponding sitespecific pain in longitudinal cohort, different pain sites were also associated with specific chronic diseases. Most pain sites were associated with an increased risk of kidney disease and digestive disease. Specifically, neck/shoulder and back pain exhibited the widest associations with chronic diseases, while unique associations were found between neck/shoulder pain and diabetes, as well as back pain and cancer (RR 2.18, 95\% CI 1.07 to 4.43 ).

The association between body pain and all-cause mortality Of the data from 17193 participants from waves 1 and 2, 15 186 participants were included in the study, with a maximum follow-up period of 2.42 years, after excluding data from participants without complete information. During the follow-up, 394 deaths were observed, of which 153 deaths of participants with body pain were identified. As presented in table 4, overall body pain was associated with elevated all-cause mortality when compared with those without any body pain (HR 1.27, 95\% CI 1.03 to 1.56). For site-specific pain, frontal torso/genital pain was associated with a $48 \%$ excess risk of all-cause mortality (HR $1.48,95 \%$ CI 1.13 to 1.93 ) when compared with those without frontal torso/genital pain. In addition, as shown in online supplemental table S4, a single pain site, but not multiple pain sites, was associated with increased all-cause mortality (HR 1.4, 95\% CI 1 to 1.96 ).

\section{Subgroup and sensitivity analysis}

Subgroup analyses were conducted according to risk factors and the potential modification effects were estimated. The results showed no potential interaction among stratified risk factors (online supplemental figure S2). To assess the robustness of the associations, we tested the association in a shorter follow-up (2011-2015). The previously identified associations remained significant except for the association with the risk of overall chronic disease (RR 1.15, 95\% CI 0.98 to 1.34 , online supplemental table S5). Furthermore, to avoid reverse causality, we excluded participants who developed the chronic disease within the 2-year follow-up (2011-2013) at baseline. Heart, kidney, and respiratory disease were no longer associated with body pain in the fully adjusted model, suggesting a potential causal inversion (online supplemental table S6). Repeated analyses were conducted through log-binomial regression to evaluate RR (online supplemental table S7) and even stronger associations were found.

\section{DISCUSSION}

To the best of our knowledge, this study is the first longitudinal prospective study a using representative, high-quality, national samples of approximately 17500 Chinese residents to explore the association between body pain and the future risk of chronic disease. We demonstrated that body pain was positively

Table 3 Unadjusted models and adjusted models estimated the association between body pain and specific diseases

\begin{tabular}{|c|c|c|c|c|c|c|c|c|c|}
\hline \multirow[b]{2}{*}{ Disease } & \multicolumn{3}{|c|}{ Model $1^{*}$} & \multicolumn{3}{|c|}{ Model 2† } & \multicolumn{3}{|c|}{ Model 3‡ } \\
\hline & Total & RR $(95 \% \mathrm{Cl})$ & $P$ value & Total & RR (95\% CI) & $P$ value & Total & RR (95\% CI) & $P$ value \\
\hline Hypertension & 5120 & $1.04(0.88$ to 1.23$)$ & 0.62 & 5110 & $1.02(0.86$ to 1.2$)$ & 0.85 & 4365 & $1.02(0.85$ to 1.23$)$ & 0.82 \\
\hline Dyslipidemia & 5212 & 1.14 (0.92 to 1.42$)$ & 0.22 & 5202 & 1.21 (0.98 to 1.51$)$ & 0.08 & 4446 & $1.15(0.9$ to 1.46$)$ & 0.26 \\
\hline Diabetes & 5229 & 1.3 (0.99 to 1.7$)$ & 0.06 & 5219 & 1.33 (1.01 to 1.76$)$ & 0.04 & 4458 & 1.16 (0.86 to 1.58$)$ & 0.33 \\
\hline Cancer & 5254 & 0.92 (0.48 to 1.74$)$ & 0.79 & 5244 & $0.98(0.51$ to 1.89$)$ & 0.96 & 4476 & 1.28 (0.65 to 2.55$)$ & 0.48 \\
\hline Liver disease & 5247 & $1.06(0.7$ to 1.59$)$ & 0.79 & 5238 & 1.17 (0.77 to 1.77$)$ & 0.45 & 4472 & $1.17(0.74$ to 1.85$)$ & 0.49 \\
\hline Heart disease & 5204 & 1.61 (1.27 to 2.02 ) & $<0.001$ & 5195 & 1.57 (1.24 to 1.99$)$ & $<0.001$ & 4434 & 1.45 (1.12 to 1.89$)$ & $<0.01$ \\
\hline Stroke & 5249 & $1.4(1$ to 1.97$)$ & 0.04 & 5239 & 1.44 (1.02 to 2.03$)$ & 0.04 & 4471 & 1.37 (0.93 to 2.01$)$ & 0.11 \\
\hline Kidney disease & 5242 & 1.58 (1.15 to 2.16$)$ & $<0.01$ & 5232 & 1.73 (1.25 to 2.38 ) & 0.001 & 4464 & $1.83(1.28$ to 2.6$)$ & $<0.01$ \\
\hline Digestive disease & 5071 & 1.66 (1.35 to 2.04$)$ & $<0.001$ & 5061 & 1.57 (1.27 to 1.95$)$ & $<0.001$ & 4331 & 1.48 (1.17 to 1.88$)$ & $<0.01$ \\
\hline Respiratory disease & 5259 & 1.54 (1.19 to 1.99$)$ & $<0.01$ & 5249 & $1.56(1.2$ to 2.03$)$ & 0.001 & 4480 & 1.43 (1.06 to 1.92$)$ & 0.02 \\
\hline
\end{tabular}

The reference group for each disease was participants without any pain.

Values with statistically significant differences have been bolded.

*Univariate model.

†Additionally adjusted for gender, age at recruitment, marital status, and educational level.

¥Additionally adjusted for BMI, sleep quality, difficulty in activity, smoking status, and alcohol consumption status.

$B M I$, body mass index; RR, risk ratio. 


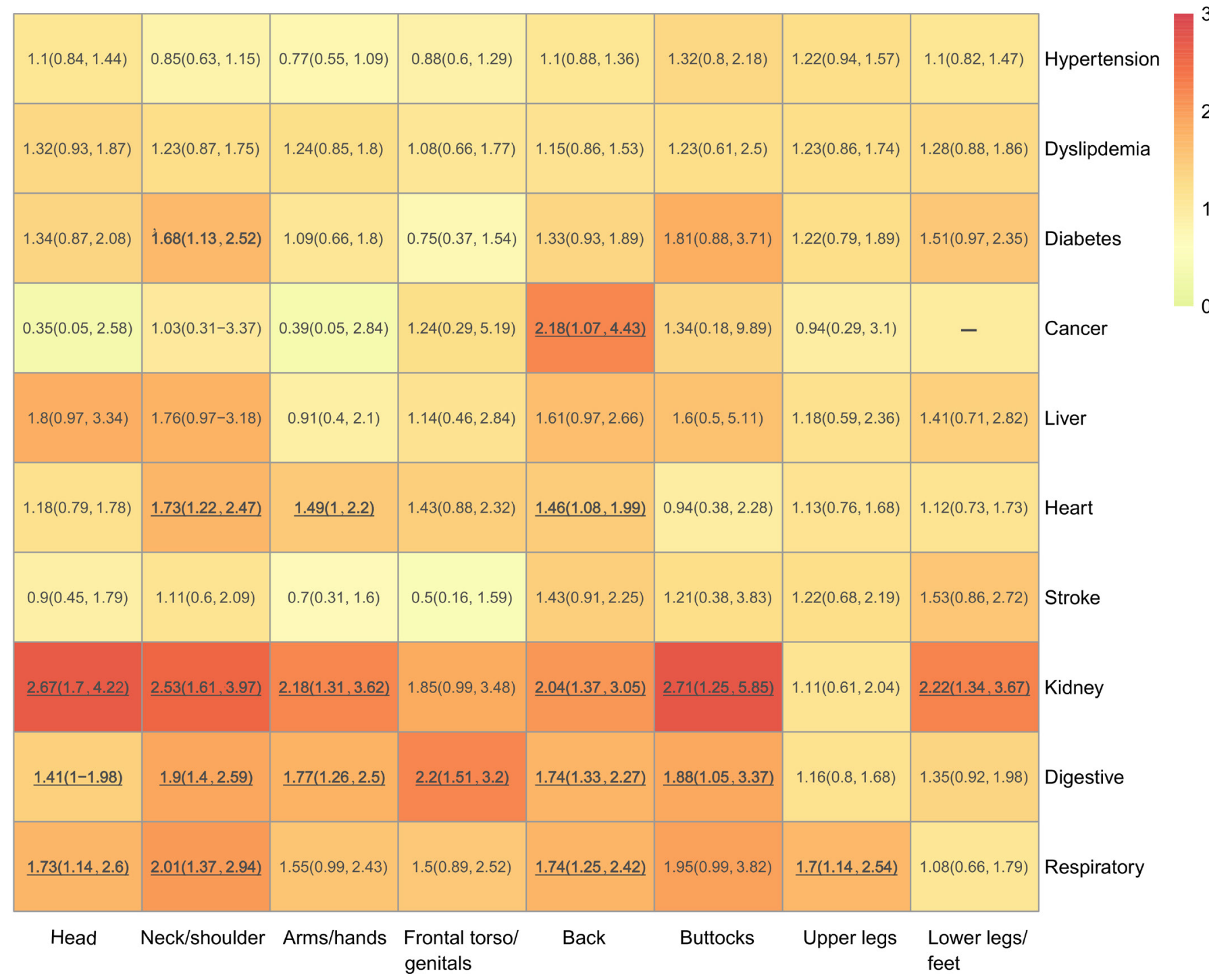

Figure 1 The fully adjusted models estimated the association between independent body pain sites and the risk of 10 specific chronic diseases in the longitudinal cohort from 2011 to 2018 using risk ratios with their $95 \% \mathrm{Cl}$. The reference group of each site-specific pain was participants without the corresponding site-specific pain (eg, the reference group of head pain was participants without head pain). Models were adjusted for gender, age at recruitment, marital status, educational level, body mass index, sleep quality, difficulty in activity, smoking status, and alcohol consumption status.

associated with future risk of chronic disease development, particularly with respiratory, heart, kidney, and digestive disease.

In a cross-sectional setting, the present study revealed that overall body pain and site-specific body pain were associated with the development of most of the diseases in elderly Chinese people. The same broad association between pain and the development of different diseases has been observed in other cohorts. ${ }^{12} 13$ Although the pain-disease association seems affirmative, the cross-sectional study design inhibited the further illustration of chronological order.

Multiple longitudinal cohorts have assessed the associations of body pain with future risk of different diseases and a positive relationship between the two has been revealed. Chronic widespread pain was also related to a positive association between cancer, cardiovascular, respiratory, and other disease-related causes, according to the evidence from half a million prospective cohort and meta-analysis. ${ }^{7}$ However, these studies have mostly focused on individual body pain site or specific outcomes, and their measurements of pain vary. ${ }^{14} 15$ Multiple waves and the wide ranges of inquired information in CHARLS allowed us to measure this association over a 7-year follow-up. By applying a single indicator of body pain and associating it with all-cause mortality and overall chronic disease risks, we found that overall body pain was associated with all-cause mortality and overall chronic disease risk. When we used site-specific pain as a more precise and individual reflection of pain, our results demonstrated that each pain site was related to the varied risk of different diseases. Although some of these associations have been revealed by previous studies such as diabetes ${ }^{9}$ and heart disease ${ }^{16}$ several associations were first identified in our study.

How body pain contributes to future chronic disease development has been explored in several studies. Evidence has shown that pain and pain-induced disability may limit physical activities or result in poor sleep quality, which may cause obesity or other mediators in heart disease or diabetes. ${ }^{17}$ In our study, the association between body pain and diabetes was largely attenuated after additional adjustment for BMI and physical conditions, which were partly confirmed previous results and suggested the potential mediator effects between them. However, for most other diseases, including heart, kidney, and digestive disease, 
Table 4 Unadjusted models and adjusted models estimated the association between overall and site-specific pain and all-cause mortality

\begin{tabular}{lllll}
\hline & HR $(95 \% \mathrm{Cl})^{*}$ & & & \\
\cline { 2 - 5 } Pain sites & Model $1 \dagger$ & P value & Model $2 \ddagger$ & P value \\
\hline Overall & $1.31(1.07$ to 1.6$)$ & 0.01 & $1.27(1.03$ to 1.56$)$ & 0.02 \\
\hline Head & $1.11(0.84$ to 1.46$)$ & 0.47 & $1.15(0.86$ to 1.52$)$ & 0.34 \\
\hline Neck/shoulder & $1.06(0.8$ to 1.39$)$ & 0.68 & $1.12(0.84$ to 1.47$)$ & 0.44 \\
\hline Arms/hands & $1.15(0.87$ to 1.51$)$ & 0.33 & $1.17(0.89$ to 1.55$)$ & 0.26 \\
\hline Frontal torso/genitals & $1.5(1.15$ to 1.95$)$ & $<0.01$ & $1.48(1.13$ to 1.93$)$ & $<0.01$ \\
\hline Back & $1.13(0.89$ to 1.42$)$ & 0.31 & $1.14(0.9$ to 1.44$)$ & 0.29 \\
\hline Buttocks & $1.4(0.9$ to 2.17$)$ & 0.14 & $1.26(0.81$ to 1.96$)$ & 0.30 \\
\hline Upper legs & $1.46(1.14$ to 1.86$)$ & $<0.01$ & $1.25(0.97$ to 1.6$)$ & 0.08 \\
\hline Lower legs/feet & $0.96(0.73$ to 1.28$)$ & 0.80 & $0.94(0.71$ to 1.25$)$ & 0.69 \\
\hline
\end{tabular}

${ }^{*}$ For overall pain, participants without any pain were set as the reference group. For any site-specific pain, participants without the corresponding site-specific pain were set as the reference group (eg, participants without head pain were set as a reference group when models were constructed to estimate the HR of head pain). tUnivariate model.

$\ddagger$ Additionally adjusted for gender, age at recruitment, marital status, and educational level.

associations were found with previous body pain, which was independent of BMI and physical condition. Thus, other potential causal pathways may exist between early detected pathways and disease. Although further studies are warranted, one potential explanation of this association could be the usage of prescription medications. Studies have demonstrated that adults in the USA with lower back pain were more likely to use prescription pain medications, hypnotics, and antidepressants (OR ranged from 1.95 for hypnotics to 6.80 for gabapentinoids). ${ }^{18}$ The overdose and/or long-term use of prescription medications, such as opioids and non-steroidal anti-inflammatory drugs, could cause severe myocardial infarction and other gastrointestinal and renal complications. $^{1920}$

The present study supports that body pain exerts a long-term influence on the development of chronic disease and several strengths of this study substantiate the evidence. First, information on body pain, pain sites, and the number of pain instances was obtained across 28 provinces, 150 districts, and 450 communities in China through systematic sampling. The sampling results represent the Chinese cohort well and enable us to infer the global status of body pain in the Chinese population. ${ }^{10}$ Second, the outcome-wide analysis was used to analyze the potential associations. The outcome-wide approach, compared with exposure-wide studies that use a single disease as an outcome, has been advocated in population studies as resulting in lower investigation bias, good comparability between effective size, and more methodological effectiveness. ${ }^{21}$

Several limitations exist in the present study. First, although we adjusted a wide range of potential confounders, residual confounding was unavoidable. Since the results we demonstrated were based on an elderly Chinese population, whether the association can be generalized to other ethnicities warrants further investigation. Additionally, the lack of detailed pain frequency and duration restrict us from further explaining these associations. Further studies that evaluated the pain intensity with a validated functional scale are warranted on this topic. Although we used a prospective cohort study to explore the associations in a chronological order, sensitivity analyses suggested that the potential causal inversion may have occurred. Moreover, given that the RRs reported in the present study were relatively lower, the possible causative relationships between body pain and chronic diseases still need further exploration. ${ }^{22} 23$ In addition, all-cause mortality was only estimated in a relatively shorter follow-up because of the lack of released data in CHARLS, leading to less exact estimates. Also, the limited sample size on mortality data restrains us to further analyze between body pain and disease-specific mortality.

In conclusion, by using an outcome-wide approach in a large population-based prospective cohort in China, the present study revealed the association between body pain and the risk of future chronic disease. Different pain sites were associated with the development of various diseases, among which back pain was associated with most chronic diseases. On the clinical level, selfreported body pain in elderly people might help us identify those at risk of future chronic disease. Considering the prevalence and global burden of body pain, effective pain management might have a considerably beneficial impact on chronic disease development at a population level.

\section{Author affiliations}

${ }^{1}$ Department of Epidemiology and Biostatistics, West China School of Public Health and West China Fourth Hospital/National Clinical Research Center for Geriatrics, West China Hospital, Sichuan University, Chengdu, Sichuan, China

${ }^{2}$ Department of Biotherapy, National Clinical Research Center for Geriatrics, Cancer Center, West China Hospital, Sichuan University, Chengdu, Sichuan, China

${ }^{3}$ Department of Nursing, National Clinical Research Center for Geriatrics, West China Hospital, Sichuan University, Chengdu, Sichuan, China

${ }^{4}$ Department of Epidemiology and Biostatistics, West China School of Public Health and West China Fourth Hospital, Sichuan University, Chengdu, Sichuan, China

Acknowledgements The authors thank the China Health and Retirement Longitudinal Study team, the National School of Development, and Institute of Social Science Survey at Peking University for providing data and training in using the datasets. The authors thank the students who participated in the survey for their cooperation. The authors thank all volunteers and staff involved in this research.

Contributors XP and ZL conceived the idea for the study. XH, QZ, and YL designed the study methodology. $L Y$ and $J L$ accessed and validated the dataset. YL carried out the formal data analysis. ZL and YL interpreted the data. ZL wrote the first draft of the report. $\mathrm{XH}, \mathrm{XP}$, and $\mathrm{QZ}$ provided advice on the first draft and revised the report critically for important intellectual content. All authors reviewed and had final approval of the submitted and published versions.

Funding The work was supported by the National Natural Science Foundation of China (81672386 and 81803104), the Sichuan Province Science and Technology Support Program (No. 2021YFSY008), and the technology Innovation Project of Chengdu Science and Technology Bureau (No. 2019-YF05-00459-SN).

Competing interests None declared.

Patient consent for publication Not required.

Ethics approval Ethical approval for all the China Health and Retirement Longitudinal Study waves was granted from the Institutional Review Board (IRB) at Peking University. The IRB approval number for the main household survey, including anthropometrics, is IRB00001052-11015.

Provenance and peer review Not commissioned; externally peer reviewed.

Data availability statement Data are available in a public, open access repository. The China Health and Retirement Longitudinal Study (CHARLS) data are freely available to researchers through the CHARLS official website at: http://charls. pku.edu.cn/.

\section{REFERENCES}

1 Fayaz A, Croft P, Langford RM, et al. Prevalence of chronic pain in the UK: a systematic review and meta-analysis of population studies. BMJ Open 2016;6:e010364.

2 IASP Terminology - IASP. Available: https://www.iasp-pain.org/Education/Content. aspx? ItemNumber=1698\#Pain [Accessed 17 Jan 2021].

3 Mills SEE, Nicolson KP, Smith BH. Chronic pain: a review of its epidemiology and associated factors in population-based studies. Br J Anaesth 2019;123:e273-83.

4 GBD 2016 Disease and Injury Incidence and Prevalence Collaborators. Global, regional, and national incidence, prevalence, and years lived with disability for 328 
diseases and injuries for 195 countries, 1990-2016: a systematic analysis for the global burden of disease study 2016. Lancet 2017;390:1211-59.

5 Siddall PJ, Cousins MJ. Persistent pain as a disease entity: implications for clinical management. Anesth Analg 2004;99:510-20.

6 Torrance N, Elliott AM, Lee AJ, et al. Severe chronic pain is associated with increased 10 year mortality. A cohort record linkage study. Eur J Pain 2010;14:380-6.

7 Macfarlane GJ, Barnish MS, Jones GT. Persons with chronic widespread pain experience excess mortality: Iongitudinal results from UK Biobank and meta-analysis. Ann Rheum Dis 2017;76:1815-22.

8 Williams A, Kamper SJ, Wiggers JH, et al. Musculoskeletal conditions may increase the risk of chronic disease: a systematic review and meta-analysis of cohort studies. BMC Med 2018;16:1-9.

9 Heuch I, Heuch I, Hagen K, et al. Is chronic low back pain a risk factor for diabetes? the Nord-Trøndelag health study. BMJ Open Diabetes Res Care 2018;6:e000569-8.

10 Zhao Y, Hu Y, Smith JP, et al. Cohort profile: the China health and retirement longitudinal study (CHARLS). Int J Epidemiol 2014;43:61-8.

11 Widerström-Noga E, Biering-Sørensen F, Bryce T, et al. The International spinal cord injury pain basic data set. Spinal Cord 2008;46:818-23.

12 Rambod M, Forsyth K, Sharif F, et al. Assessment and management of pain in children and adolescents with bleeding disorders: a cross-sectional study from three haemophilia centres. Haemophilia 2016;22:65-71.

13 Lee YC, Bingham CO, Edwards RR, et al. Association between pain sensitization and disease activity in patients with rheumatoid arthritis: a cross-sectional study. Arthritis Care Res 2018:70:197-204.

$14 \mathrm{Hsu}$ H-J, Wu I-W, Hsu K-H, et al. The association between chronic musculoskeletal pain and clinical outcome in chronic kidney disease patients: a prospective cohort study. Ren Fail 2019;41:257-66
15 Battaglia M, Garon-Carrier G, Brendgen M, et al. Trajectories of pain and anxiety in a longitudinal cohort of adolescent twins. Depress Anxiety 2020;37:475-84.

16 Zhu K, Devine A, Dick IM, et al. Association of back pain frequency with mortality, coronary heart events, mobility, and quality of life in elderly women. Spine 2007;32:2012-8 https://journals.Iww.com/spinejournal/Fulltext/2007/08150/ Association_of_Back_Pain_Frequency_With_Mortality,.15.aspx

17 van der Zee-Neuen A, Putrik P, Ramiro S, et al. Impact of chronic diseases and multimorbidity on health and health care costs: the additional role of musculoskeletal disorders. Arthritis Care Res 2016;68:1823-31.

18 Shmagel A, Ngo L, Ensrud K, et al. Prescription medication use among communitybased U.S. adults with chronic low back pain: a cross-sectional population based study. J Pain 2018;19:1104-12.

19 Gouveia N, Rodrigues A, Ramiro S, et al. The use of analgesic and other Pain-Relief drugs to manage chronic low back pain: results from a national survey. Pain Pract 2017; 17:353-65.

20 Enthoven WTM, Roelofs PDDM, Deyo RA, et al. Non-Steroidal anti-inflammatory drugs for chronic low back pain. Cochrane Database Syst Rev 2016;2:CD012087.

21 VanderWeele TJ, Mathur MB, Chen Y. Outcome-wide longitudinal designs for causal inference: a new template for empirical studies. arXiv 2018.

22 Boffetta P. Causation in the presence of weak associations. Crit Rev Food Sci Nutr 2010:50:13-16.

23 Haddock CK, Rindskopf D, Shadish WR. Using odds ratios as effect sizes for metaanalysis of dichotomous data: a primer on methods and issues. Psychol Methods 1998;3:339-53. 